\title{
Two-Stage Intuitionistic Fuzzy Transportation Problem through the Prism of Index Matrices
}

\author{
Velichka Traneva \\ "Prof. Asen Zlatarov" University \\ "Prof. Yakimov" Blvd, Burgas 8000, Bulgaria \\ Email: veleka13@gmail.com
}

\author{
Stoyan Tranev \\ "Prof. Asen Zlatarov" University \\ "Prof. Yakimov" Blvd, Burgas 8000, Bulgaria \\ Email: tranev@abv.bg
}

\begin{abstract}
In today's market environment not all the parameters of the transportation problems may not be known precisely. Uncertain data can be represented by fuzzy sets (FSs). Intuitionistic FSs (IFSs) are an extension of FSs with a degree of hesitancy. The paper presents a new approach for solution of a two-stage intuitionistic fuzzy transportation problem (2-S IFTP) through the prism of index matrices (IMs). Its main objective is to find the quantities of delivery from manufacturers and resselers to buyers to maintain the supply and demand requirements at the cheapest transportation costs. The solution procedure is demonstrated by a numerical example.
\end{abstract}

\section{INTRODUCTION}

T HE TP originally was proposed by Hitchcock in 1941 [14].

In conventional $\mathrm{TP}$, values of the transportation cost, the demanded and offered quantities of the product are precisely defined. In real-life TPs, some of their parameters are uncertain due to climatic, road conditions or other market conditions. In some TPs, destinations cannot get all the required quantity of product due to limited storage capacity. In this case, the necessary quantities of products are sent to the destinations in two stages. Initially, the minimum destination requirements are sent from the sources to the destinations. Once part of the entire initial shipment has been used up, they are ready to receive the remaining quantity in the second stage. This type of transportation problem is known as two-stage TPs (2-S TPs). The main purpose of the 2-S TP is to transport the items from the origins to the destinations in two stages such way that the total transportation costs in the two stages are minimum [43]. In real life 2-S TPs, information about the parameters of the problem is uncertain due to weather and road conditions, lack of good communications, traffic jams, etc. For description of imprecise information, Zadeh has developed the theory of fuzzy sets (FSs) [29]. An extension of FSs is intuitionistic fuzzy sets (IFSs), which was proposed by Atanassov in 1983 [18]. The main difference between FSs and IFSs is that the IFSs have a degree of hesitancy.

Let us give a brief literature overview of the works on the topic fuzzy (FTPs) and intuitionistic fuzzy transportation

Work on Sect. I and Sect. II is supported by the Asen Zlatarov University through project Ref. No. NIX-440/2020 "Index matrices as a tool for knowledge extraction". Work on Sect. III and Sect. IV is supported by the Ministry of Education and Science under the Programme "Young scientist and postdoctoral students", approved by DCM \# 577/17.08.2018. problems (IFTPs). Chanas et al., in 1984, has proposed a fuzzy linear programming model for solving TPs with clear transportation costs, fuzzy supply and demand values [39]. Jimenez and Verdegay, in 1999, researched fuzzy Solid TP with trapezoidal FNs and presented a genetic approach for solving FTP [13]. Liu and Kao [41] have demonstrated a method, based on Zadeh's extension principle, to find the optimal solution of the trapezoidal FTPs. Dinagar and Palanivel [11] have described a fuzzy modified distribution method to find the fuzzy optimal solution of FTPs in which all the parameters are represented by trapezoidal fuzzy numbers. Pandian and Natarajan, in 2010, developed zero-point method for solution for FTP with trapezoidal fuzzy parameters [34]. In [1] was proposed a new method based on fuzzy zero-point method for finding a more-or-less fuzzy optimal solution for such FTPs in which all the parameters are represented by trapezoidal fuzzy numbers.

Kaur and Kumar, in 2012, introduced fuzzy least cost method, fuzzy north west corner rule and fuzzy Vogel approximation method for determining of an optimal solution of FTP [33]. Basirzadeh [17] has found a fuzzy optimal solution of fully FTPs by transforming the fuzzy parameters into the crisp parameters using classical algorithms. Gani et al. [2] used Arsham and Khan's simplex algorithm [16] to find a fuzzy optimal solution of FTPs with trapezoidal fuzzy parameters. Patil and Chandgude, in 2012, performed "Fuzzy Hungarian approach" for TP with trapezoidal FNs [7]. A modified Vogel's approximation method for finding an optimal solution of FTPs was proposed in [8]. Aggarwal and Gupta, in 2013, described a procedure for solving intuitionistic fuzzy TP (IFTP) with trapezoidal IFNs via ranking method [15]. Jahihussain and Jayaraman, in 2013, presented a zerosuffix method for obtaining an optimal solution for FTPs with triangular and trapezoidal FNs (see [36], [37]). Zero suffix method to solve FTP after its converting into the crisp problem was applied in [32] and [44]. A fuzzified version of zero suffix method was performed and applied in [30], in 2018, to FTPs. Shanmugasundari and Ganesan, in 2013, proposed a fuzzy modified distribution algorithm and a fuzzy approximation method of Vogel to solve FTP with FNs [31]. Gani and Abbas, in 2014 [4], and Kathirvel, and Balamurugun, in 2012 (see [27], [28]), proposed a method for solving TP in which the quantities demanded and offered are represented in the form 
of the trapezoidal intuitionistic FNs (IFNs). In well-known and commonly used methods, proposed by Basirzadeh [17], Gani et al. [2], Pandian and Natarajan [34] and Dinagar and Palanivel [11], there is a problem that, in a general case, neither the cost values, nor the obtained fuzzy optimal solution need necessarily to be non-negative fuzzy numbers. These are shortcomings of these methods, as in real life problems there is no physical meaning of a negative value of the cost and a negative quantity of the product transported. In [41] was developed a method for solution fully FTPs with both the inequality and equality constraints in which all the parameters are represented by non-negative trapezoidal fuzzy numbers. Fully FTPs was resolved in [40], in 2017, using a new method, based on the Hungarian and MODI algorithm. The methods for finding a fuzzy optimal solution of TPs with the LR flat fuzzy numbers were proposed in [6], based on the tabular representation and on the fuzzy linear programming formulation to overcome these shortcomings. Antony et al. used Vogel's approximation method for solving triangular IFTP in 2014 [35]. Fuzzy methods of 2-S time minimizing TPs are presented in [3], [33]. The work [42] was focused on presenting an innovative study of a multi-stage multi-objective solid trapezoidal IFTP with a green supply chain network system. 2-S time minimizing TP have considered in [38] over triangular intuitionistic fuzzy (IF) numbers. Trapezoidal and triangular IFSs are special cases of IFSs.

In our previous works [46], [47], [48], [52], we have proposed for the first time an intuitionistic fuzzy modified distribution algorithm, a zero-suffix and a zero-point method to determine an optimal solution of the IFTP, interpreted by the IFSs and IMs [18], [19] concepts. The concept of index matrices was introduced to enable two matrices with different dimensions to be summed. Later, IMs concept was extended and were defined operations, relations and operators over IMs. The IMs theoretical apparatus was described in [21], [51]. Here, we propose a novel approach to the formulation and solution 2-S IFTP, in which the transportation costs, supply and demand quantities are IFPs, depending on the climatic, road conditions and economic factors. The proposed algorithm uses IMs toolkit for modeling the 2-S IFTP and for finding of its optimal solution. The advantages of the algorithm are indicated.

The remainder of this paper is as follows: Section 2 describes some initial definitions of the theories of the IMs and the IFPs. In Section 3, we formulate 2-S IFTP and propose an algorithm for its solution by the concepts of IMs and IFSs. The effectiveness of the approach is demonstrated by an example in Section 4. Section 5 drawns conclusions and outlines directions for future research.

\section{Preparatory Definitions on Intuitionistic FUZZY PAIRS AND IMS}

In this section we recall some basic definitions on intuitionistic fuzzy pairs (IFPs) from [12], [20], [22], [26], [49] and on index matrices tool from [21], [51].

\subsection{Basic Remarks on IFPs}

An IFP is under the form of an ordered pair $\langle a, b\rangle=$ $\langle\mu(p), v(p)\rangle$, where $a, b \in[0,1]$ and $a+b \leq 1$, that is used as an evaluation of a proposition $p$ [22], [26]. $\mu(p)$ and $v(p)$ respectively determine the "truth degree" (degree of membership) and "falsity degree" (degree of non-membership).

In the works [10], [12], [20], [26], [24] were proposed some basic operations over two IFPs $x=\langle a, b\rangle$ and $y=\langle c, d\rangle$ :

$$
\begin{gathered}
\neg x=\langle b, a\rangle ; \\
x \wedge_{1} y=\langle\min (a, c), \max (b, d)\rangle ; \\
x \vee_{1} y=\langle\max (a, c), \min (b, d)\rangle ; \\
x \wedge_{2} y=x+y=\langle a+c-a \cdot c, b . d\rangle ; \\
x \vee_{2} y=x \cdot y=\langle a \cdot c, b+d-b . d\rangle ; \\
\alpha . x=\left\langle 1-(1-a)^{\alpha}, b^{\alpha}\right\rangle(\text { for } \alpha=n \text { or } 1 / n(n \in N)) ; \\
x-y=\langle\max (0, a-c), \min (1, b+d, 1-a+c)\rangle \\
x: y=\left\{\begin{array}{c}
\langle\min (1, a / c), \min (\max (0,1-a / c), \\
\max (0,(b-d) /(1-d))) \text { if } c \neq 0 \& d \neq 1 \\
\langle 0,1\rangle \text { otherwise }
\end{array}\right.
\end{gathered}
$$

The forms of the relations with IFPs are the following

$$
\begin{array}{cc}
x \geq y \text { iff } a \geq c \text { and } b \leq d ; & x \leq y \text { iff } a \leq c \text { and } b \geq d ; \\
x \geq_{\square} y \text { iff } a \geq c ; & x \leq_{\square} y \text { iff } a \leq c ; \\
x \geq_{\diamond} y \text { iff } b \leq d ; & x \leq_{\diamond} y \text { iff } b \geq d ; \\
x=y & \text { iff } a=c \text { and } b=d \\
x \geq_{R} y & \text { iff } R_{\langle a, b\rangle} \leq R_{\langle c, d\rangle},
\end{array}
$$

where $R_{\langle a, b\rangle}=0.5(2-a-b)(1-a)[12]$.

The IFP $x$ is an "intuitionistic fuzzy false pair" (IFFP) if and only if $a \leq b$.

2.2. Definition, Operations and Relations over Intuitionistic Fuzzy Index Matrices

One of the basic IM-types are intuitionistic fuzzy IMs (IFIMs) whose elements are IFPs. Let $\mathscr{I}$ be a fixed set. The definition of two-dimensional IFIM (2-D IFIM) $\left[K, L,\left\{\left\langle\mu_{k_{i}, l_{j}}, v_{k_{i}, l_{j}}\right\rangle\right\}\right]$ with index sets $K$ and $L \quad(K, L \subset \mathscr{I})$ is the following: \begin{tabular}{c|ccccc} 
& $l_{1}$ & $\ldots$ & $l_{j}$ & $\ldots$ & $l_{n}$ \\
\hline$k_{1}$ & $\left\langle\mu_{k_{1}, l_{1}}, v_{k_{1}, l_{1}}\right\rangle$ & $\ldots$ & $\left\langle\mu_{k_{1}, l_{j}}, v_{k_{1}, l_{j}}\right\rangle$ & $\ldots$ & $\left\langle\mu_{k_{1}, l_{n}}, v_{k_{1}, l_{n}}\right\rangle$ \\
$\vdots$ & $\vdots$ & $\ddots$ & $\vdots$ & $\ddots$ & $\vdots$ \\
$k_{m}$ & $\left\langle\mu_{k_{m}, l_{1}}, v_{k_{m}, l_{1}}\right\rangle$ & $\ldots$ & $\left\langle\mu_{k_{m}, l_{j}}, v_{k_{m}, l_{j}}\right\rangle$ & $\ldots$ & $\left\langle\mu_{k_{m}, l_{n}}, v_{k_{m}, l_{n}}\right\rangle$
\end{tabular}

where for $i=1, \ldots, m ; j=1, \ldots, n$ :

$$
0 \leq \mu_{k_{i}, l_{j}}, v_{k_{i}, l_{j}}, \mu_{k_{i}, l_{j}}+v_{k_{i}, l_{j}} \leq 1
$$

The basic operations over two IMs

$$
A=\left[K, L,\left\{\left\langle\mu_{k_{i}, l_{j}}, v_{k_{i}, l_{j}}\right\rangle\right\}\right] \text { and } B=\left[P, Q,\left\{\left\langle\rho_{p_{r}, q_{s}}, \sigma_{p_{r}, q_{s}}\right\rangle\right\}\right]
$$

are as follows [21]:

Negation: $\neg A=\left[K, L,\left\{\left\langle v_{k_{i}, l_{j}}, \mu_{k_{i}, l_{j}}\right\rangle\right\}\right]$. 
Addition-(o,*): $A \oplus_{(\circ, *)} B=\left[K \cup P, L \cup Q,\left\{\left\langle\phi_{t_{u}, v_{w}}, \psi_{t_{u}, v_{w}}\right\rangle\right\}\right]$, where $\left\langle\phi_{t_{u}, v_{w}}, \psi_{t_{u}, v_{w}}\right\rangle$

$$
= \begin{cases}\left\langle\mu_{k_{i}, l_{j}}, v_{k_{i}, l_{j}}\right\rangle, & \text { if } t_{u}=k_{i} \in K \text { and } v_{w}=l_{j} \in L-Q \\ \left\langle\rho_{p_{r}, q_{s}}, \sigma_{p_{r}, q_{s}}\right\rangle, & \text { if } t_{u}=k_{r} \in K-P \text { and } v_{w}=l_{j} \in L ; \\ & \text { or } t_{u}=p_{r} \in P-K \\ & \text { and } v_{w}=q_{s} \in Q-L \\ \left\langle\circ\left(\mu_{k_{i}, l_{j}}, \rho_{p_{r}, q_{s}}\right),\right. & \text { if } t_{u}=k_{i}=p_{r} \in K \cap P \\ \left.*\left(v_{k_{i}, l_{j}}, \sigma_{p_{r}, q_{s}}\right)\right\rangle, & \text { and } v_{w}=l_{j}=q_{s} \in L \cap Q ; \\ \langle 0,1\rangle, & \text { otherwise. }\end{cases}
$$

where $\langle\circ, *\rangle \in\{\langle\max , \min \rangle,\langle\min , \max \rangle,\langle$ average, average $\rangle\}$. Termwise subtraction-(max,min):

$$
A-_{(\max , \min )} B=A \oplus_{(\max , \min )} \neg B .
$$

Termwise multiplication- $(\min , \max )$ :

$$
A \otimes_{(\min , \max )} B=\left[K \cap P, L \cap Q,\left\{\left\langle\phi_{t_{u}, v_{w}}, \psi_{t_{u}, v_{w}}\right\rangle\right\}\right],
$$

where

$$
\left\langle\phi_{t_{u}, v_{w}}, \psi_{t_{u}, v_{w}}\right\rangle=\left\langle\min \left(\mu_{k_{i}, l_{j}}, \rho_{p_{r}, q_{s}}\right), \max \left(v_{k_{i}, l_{j}}, \sigma_{p_{r}, q_{s}}\right)\right\rangle .
$$

\section{Multiplication:}

$$
A \odot_{(\circ, *)} B=\left[K \cup(P-L), Q \cup(L-P)\left\{\left\langle\phi_{t_{u}, v_{w}}, \psi_{t_{u}, v_{w}}\right\rangle\right\}\right],
$$

where $\left\langle\phi_{t_{u}, v_{w}}, \psi_{t_{u}, v_{w}}\right\rangle$ is defined in [21] and $\langle 0, *\rangle \in$ $\left\{\langle\max , \min \rangle,\langle\min , \max \rangle,\left\langle\wedge_{2}, \vee_{2}\right\rangle\right\}$.

Transposition: $A^{T}$ is the transposed IM of $A$.

Reduction: The symbol " $\perp$ " denotes the lack of some component in the definitions. The operation $(k, \perp)$-reduction of the IM $A$ is defined by: $A_{(k, \perp)}=\left[K-\{k\}, L,\left\{c_{t_{u}, v_{w}}\right\}\right]$,

where $c_{t_{u}, v_{w}}=a_{k_{i}, l_{j}}$ for $t_{u}=k_{i} \in K-\{k\}$ and $v_{w}=l_{j} \in L$.

Projection: Let $M \subseteq K$ and $N \subseteq L$. Then,

$$
\operatorname{pr}_{M, N} A=\left[M, N,\left\{b_{k_{i}, l_{j}}\right\}\right],
$$

where for each $k_{i} \in M$ and each $l_{j} \in N, b_{k_{i}, l_{j}}=a_{k_{i}, l_{j}}$.

Substitution: Let IM $A=\left[K, L,\left\{a_{k, l}\right\}\right]$ be given. Some forms of the substitution over $A$ are defined for the couples of indices $(p, k)$ by

$$
\left[\frac{p}{k} ; \perp\right] A=\left[(K-\{k\}) \cup\{p\}, L,\left\{a_{k, l}\right\}\right] .
$$

Index type operations [45]:

AGIndex $_{\left\{(\min / \max ) /\left(\min \square / \max _{\square}\right) /\left(\min _{\diamond} / \max _{\diamond}\right)\left(\min _{R} / \max _{R}\right)\right\}(\not \subset)}(A)$

$$
=\left\langle k_{i}, l_{j}\right\rangle
$$

finds the index of the minimum/ maximum element of $A$ with no empty value in accordance with the relations (3).

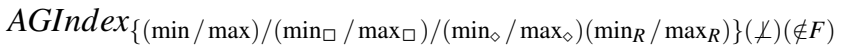

$$
(A)=\left\langle k_{i}, l_{j}\right\rangle
$$

presents the index of the minimum/ maximum element between the elements of $A$, whose indexes $\notin F$, with no empty value in accordance with the relations (3).

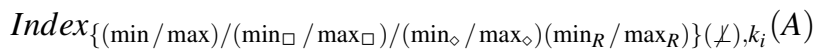

$$
=\left\{\left\langle k_{i}, l_{v_{1}}\right\rangle, \ldots,\left\langle k_{i}, l_{v_{x}}\right\rangle, \ldots,\left\langle k_{i}, l_{v_{V}}\right\rangle\right\},
$$

where $\left\langle k_{i}, l_{v_{x}}\right\rangle$ (for $i=1, \ldots, m ; j=1, \ldots, n ; x=1, \ldots, V$ ) are the indices of the minimum/ maximum IFFP of $k_{i}$-th row of $A$ with no empty value in accordance with the relations (3).

$$
\operatorname{Index}_{(\not \chi)}(A)=\left\{\left\langle k_{1}, l_{v_{1}}\right\rangle, \ldots,\left\langle k_{i}, l_{v_{i}}\right\rangle, \ldots,\left\langle k_{m}, l_{v_{m}}\right\rangle\right\},
$$

where $\left\langle k_{i}, l_{v_{i}}\right\rangle$ (for $1 \leq i \leq m$ ) are the indices of the element of $A$, whose cell is full.

\section{Aggregation operations}

Let us use the operations $\#_{q},(q \leq i \leq 3)$ from [50] for scaling aggregation operations over two IFPs $x=\langle a, b\rangle$ and $y=\langle c, d\rangle$ : $x \#_{1} y=\langle\min (a, c), \max (b, d)\rangle$;

$x \#_{2} y=\langle\operatorname{average}(a, c)$, average $(b, d)\rangle$;

$x \#_{3} y=\langle\max (a, c), \min (b, d)\rangle$.

Let $k_{0} \notin K$ be a fixed index. The definition of the aggregation operation by the dimension $K$ is [21], [50]: is:

$$
\begin{aligned}
& \alpha_{K, \#_{q}}\left(A, k_{0}\right) \\
& =\begin{array}{c|cccc} 
& & l_{1} & \ldots & \multicolumn{1}{c}{l_{n}} \\
\hline \multirow{2}{*}{k_{0}} & \begin{array}{c}
m \\
\#_{q} \\
\end{array} & \left\langle\mu_{k_{i}, l_{1}}, v_{k_{i}, l_{1}}\right\rangle & \ldots & \#_{q}^{m}\left\langle\mu_{k_{i}, l_{n}}, v_{k_{i}, l_{n}}\right\rangle
\end{array},
\end{aligned}
$$

where $1 \leq q \leq 3$.

Aggregate global internal operation [45]:

$$
A G I O_{\oplus_{(\circ, *)}}(A),
$$

where $\langle\circ, *\rangle \in\left\{\langle\max , \min \rangle,\langle\min , \max \rangle,\left\langle\wedge_{2}, \vee_{2}\right\rangle\right\}$.

Non-strict relation "inclusion about value" The form of this type of relations between two IMs $A$ and $B$ is as follows:

$$
A \subseteq_{v} B \text { iff }(K=P) \&(L=Q) \&(\forall k \in K)(\forall l \in L)\left(a_{k, l} \leq b_{k, l}\right) .
$$

\section{INTUITIONISTIC FUZZY INDEX MATRIX APPROACH TO} TwO-STAGE IFTP

Let us extend the IFTP from [48] into a two-stage one as follows:

\section{A. Generalized 2-S IFTP}

A trader supplies a product to different companies after delivery of that product from different producers in an uncertain environment. Destinations cannot get all the required quantity of product due to limited storage capacity. In this case, the necessary quantities of products are sent to the destinations in two stages. Initially, the minimum destination requirements are sent from the sources to the destinations. Once part of the entire initial shipment has been used up, they are ready to receive the remaining quantity in the second stage. The trader wants to find optimal solutions for the 2-S IFTP.

First stage A trader supplies a product to $n$ different companies (consumers) $\left\{l_{1}, \ldots, l_{j}, \ldots, l_{n}\right\}$ after delivery of that product from different $m$ manufacturers (producers) $\left\{k_{1}, \ldots, k_{i}, \ldots, k_{m}\right\}$ in quantities $c_{k_{i}, R}$ (for $1 \leq i \leq m$ ). Let the consumers (destinations) need this product in quantities of $c_{Q, l_{j}}$ (for $1 \leq j \leq n$ ). 
Let $c_{k_{i}, l_{j}}$ be the intuitionistic fuzzy cost for transporting a unit quantity of the product from the $k_{i}$-th producer to the $l_{j}$-th consumer; $x_{k_{i}, l_{j}}$ - the number of units of the product, transported from $k_{i}$-th source to $l_{j}$-th destination and $c_{p l, l_{j}}$ (for $1 \leq j \leq n)$ are limits to the transportation costs of the delivery a product from the $k_{i}$-th manufacturer to the $l_{j}$-th destination under form of IFPs.

Second stage Let some of the buyers $R S=$ $\left\{l_{1}^{*}, \ldots, l_{j^{*}}^{*}, \ldots, l_{n^{*}}^{*}\right\}(R S \subset L)$ become resellers. The resellers $\left\{l_{1}^{*}, \ldots, l_{j^{*}}^{*}, \ldots, l_{n^{*}}^{*}\right\}$ want to sell quantities of the product not only purchased, but also from own production or stocks at a surplus charge $c_{l_{j^{*}}^{*}, q^{*}}^{*}$ for a product unit to other consumers $\left\{u_{1}, \ldots, u_{g}, \ldots, u_{f}\right\}$, in quantities $c_{l^{*}}^{*}, R^{*}\left(\right.$ for $\left.1 \leq j^{*} \leq n^{*}\right)$.

Consumers need this product in an amount of $c_{Q^{*}, u_{g}}^{*}$ (for $1 \leq g \leq f$ ). Let $c_{l^{*}, u_{g}}^{*}$ (for $1 \leq j^{*} \leq n^{*}, 1 \leq g \leq f$ ) be the total cost for the purchase of one unit quantity of the product from the $l_{j^{*}}^{*}$-th reseller to $u_{g}$-th destination; $x_{l_{j^{*}}^{*}, u_{g}}$ - the number of units of the product, transported from the $l_{j^{*}}^{*}$-th reseller to $u_{g^{-}}$ th destination; $c_{l^{*}}^{*}, p u^{*},\left(\right.$ for $\left.1 \leq j^{*} \leq n^{*}\right)$ - is the price of a product unit of the $l_{j^{*}}^{*}$ th reseller; $c_{p l^{*}, u_{g}}^{*},($ for $1 \leq g \leq f)-$ upper limit of the price at which the $u_{g}$-th consumer wish to purchase the product.

For estimating the parameters of 2-S IFTP, we can use the expert approach described in detail in [20]. The experts are not sure about the transportation costs, the quantities of offered and demanded goods due to uncontrollable factors. The transportation costs are evaluated as intuitionistic fuzzy numbers after a thorough discussion, interpreted by the intuitionistic fuzzy concept. The purpose of the 2-S IFTP is to meet the requests of all users $\left\{l_{1}, \ldots, l_{j}, \ldots, l_{m}\right\}$ and $\left\{u_{1}, \ldots, u_{g}, \ldots, u_{f}\right\}$ from the two stages so that the intuitionistic fuzzy transportation cost is minimum.

\section{B. Solution of the 2-S IFTP}

The proposed algorithm for modeling of 2-S IFTP and finding of its optimal solution is based on IMs concept [21].

1) Solution of the First Stage of the 2-S IFTP: Step 1. At starting of the algorithm for solution of the 2-S IFTP, the cost IM $C[K, L]$ is created:

\begin{tabular}{c|ccccc} 
& $l_{1}$ & $\ldots$ & $l_{n}$ & $R$ & $p u$ \\
\hline$k_{1}$ & $\left\langle\mu_{k_{1}, l_{1}}, v_{k_{1}, l_{1}}\right\rangle$ & $\ldots$ & $\left\langle\mu_{k_{1}, l_{n}}, v_{k_{1}, l_{n}}\right\rangle$ & $\left\langle\mu_{k_{1}, R}, v_{k_{1}, R}\right\rangle$ & $\left\langle\mu_{k_{1}, p u}, v_{k_{1}, p u}\right\rangle$ \\
$\vdots$ & $\vdots$ & $\ddots$ & $\vdots$ & $\vdots$ & \\
$k_{m}$ & $\left\langle\mu_{k_{m}, l_{1}}, v_{k_{m}, l_{1}}\right\rangle$ & $\ldots$ & $\left\langle\mu_{k_{m}, l_{n}}, v_{k_{m}, l_{n}}\right\rangle$ & $\left\langle\mu_{k_{m}, R}, v_{k_{m}, R}\right\rangle$ & $\left\langle\mu_{k_{m}, p u}, v_{k_{m}, p u}\right\rangle$ \\
$Q$ & $\left\langle\mu_{Q, l_{1}}, v_{Q, l_{1}}\right\rangle$ & $\ldots$ & $\left\langle\mu_{Q, l_{n}}, v_{Q, l_{n}}\right\rangle$ & $\left\langle\mu_{Q, R}, v_{Q, R}\right\rangle$ & $\left\langle\mu_{Q, p u}, v_{Q, p u}\right\rangle$ \\
& $\left\langle\mu_{p l, l_{1}}, v_{p l, l_{1}}\right\rangle$ & $\ldots$ & $\left\langle\mu_{p l, l_{n}}, v_{p l, l_{n}}\right\rangle$ & $\left\langle\mu_{p l, R}, v_{p l, R}\right\rangle$ & $\left\langle\mu_{p l, p u}, v_{p l, p u}\right\rangle$ \\
$p l$ & $\left\langle\mu_{p u_{1}, l_{1}}, v_{p u_{1}, l_{1}}\right\rangle$ & $\ldots$ & $\left\langle\mu_{p u_{1}, l_{n}}, v_{p u_{1}, l_{n}}\right\rangle$ & $\left\langle\mu_{p u_{1}, R}, v_{p u_{1}, R}\right\rangle$ & $\left\langle\mu_{p u_{1}, p u}, v_{p u_{1}, p u}\right\rangle$
\end{tabular}

where $K=\left\{k_{1}, k_{2}, \ldots, k_{m}, Q, p l, p u_{1}\right\}, \quad L=$ $\left\{l_{1}, l_{2}, \ldots, l_{n}, R, p u\right\}$ and for $1 \leq i \leq m, 1 \leq j \leq n$, $\left\{c_{k_{i}, l_{j}}, c_{k_{i}, R}, c_{k_{i}, p u}, c_{p l, l_{j}}, c_{p l, R}, c_{p l, p u}, c_{Q, l_{j}}, c_{Q, R}, c_{Q, p u}, c_{p u_{1}, l_{j}}\right.$, $\left.c_{p u_{1}, R}, c_{p u_{1}, p u}\right\}$ are IFPs.

Let we denote by $|K|=m+3$ the number of elements of the set $K$; then $|L|=n+2$.
We also define the IM

$$
X\left[K^{I}, L^{I}\right]=\begin{array}{c|ccccc} 
& l_{1} & \ldots & l_{j} & \ldots & l_{n} \\
\hline k_{1} & x_{k_{1}, l_{1}} & \cdots & x_{k_{1}, l_{j}} & \cdots & x_{k_{1}, l_{n}} \\
\vdots & \vdots & \ddots & \vdots & \ddots & \vdots \\
k_{m} & x_{k_{m}, l_{1}} & \ldots & x_{k_{m}, l_{j}} & \ldots & x_{k_{m}, l_{n}}
\end{array},
$$

$K^{I}=\left\{k_{1}, k_{2}, \ldots, k_{m}\right\}, K^{I}=\left\{l_{1}, l_{2}, \ldots, l_{n}\right\}$, and for $1 \leq i \leq m$, $1 \leq j \leq n: x_{k_{i}, l_{j}}=\left\langle\rho_{k_{i}, l_{j}}, \sigma_{k_{i}, l_{j}}\right\rangle$. Go to Step 2 .

Step 2. For solving the first stage on the 2-S IFTP we can apply one of the algorithms, outlined in our papers [47], [48], [52]. In the program code of the developed algorithms was used a part of Microsoft Visual Studio.NET 2010 C project's.

After an application of the algorithm for finding an optimal solution of IFTP, the following conditions are checked: $D=$ Index $\chi X=\left\{\left\langle k_{i *_{1}}, l_{j *_{1}}\right\rangle, \ldots,\left\langle k_{i *_{f}}, l_{j *_{f}}\right\rangle, \ldots,\left\langle k_{i *_{\varphi}}, l_{j *_{\varphi}}\right\rangle\right\}$.

If the intuitionistic fuzzy feasible solution is degenerated (it contains less than $m+n-1$ (the total number of producers and consumers decreased by 1 ) occupied cells in the $X$ i.e. $|D|<m+n-1)$ [9] then increase the basic cells $x_{k_{i}, l_{j}}$ with one to which the minimum transportation cost corresponds. Let us the recorded delivery of this cell is $\langle 0,1\rangle$. The IMs operations are:

If

$$
|D|<m+n-1, \text { then }
$$

$\left\{\right.$ AGIndex $\left.\left._{\{(\min / \max ) /(\min \square} / \max _{\square}\right) /\left(\min _{\diamond} / \max _{\diamond}\right)\left(\min _{R} / \max _{R}\right)\right\}(\not \chi)(\notin D)(C)$

$$
\left.=\left\langle k_{\alpha}, l_{\beta}\right\rangle ; x_{k_{a l}, l_{\beta}}=\langle 0,1\rangle\right\} \text {. }
$$

for $i=1$ to $m$

for $j=1$ to $n$

If $x_{k_{i}, l_{j}}=\langle\perp, \perp\rangle$ then $x_{k_{i}, l_{j}}=\langle 0,1\rangle$.

Go to Step 3 .

Step 3. The optimal intuitionistic fuzzy transportation cost at the first stage is calculated by:

$$
A G I O_{\left.\oplus_{(\max , \min )}\right)}^{1}\left(C_{\left(\left\{Q, p l, p u_{1}\right\},\{R, p u\}\right)} \otimes_{(\min , \max )} X_{o p t}\right)
$$

or $A G I O_{\left.\oplus_{\left(\wedge_{2}\right)}\right)}^{2}\left(C_{\left(\left\{Q, p l, p u_{1}\right\},\{R, p u\}\right)} \otimes_{\left(\vee_{2}\right)} X_{o p t}\right)$,

where $\vee_{2}$ and $\wedge_{2}$ are the operations from (1).

2) Solution of the Second Stage of the 2-S IFTP: To find the optimal solution for the second stage of the problem, we propose the following algorithm, described by a program code, which is a part of Microsoft Visual Studio.NET 2010 C project.

Step 4. Let us create the following cost IFIM $C^{*}\left[L^{*}, U\right]$

\begin{tabular}{c|cccccc} 
& $u_{1}$ & $\cdots$ & $u_{f}$ & $R^{*}$ & $q^{*}$ & $p u^{*}$ \\
\hline$l_{1}^{*}$ & $c_{l_{1}^{*}, u_{1}}^{*}$ & $\cdots$ & $c_{l_{1}^{*}, u_{f}}^{*}$ & $c_{l_{1}^{*}, R^{*}}^{*}$ & $c_{l_{1}^{*}, q^{*}}^{*}$ & $c_{l_{1}^{*}, p u^{*}}^{*}$ \\
$\vdots$ & $\vdots$ & $\cdots$ & $\vdots$ & $\cdots$ & $\vdots$ & $\vdots$ \\
$l_{j^{*}}^{*}$ & $c_{l_{j^{*}}^{*}, u_{1}}^{*}$ & $\cdots$ & $c_{l_{j^{*}}^{*}, u_{f}}^{*}$ & $c_{l_{j^{*}}^{*}, R^{*}}$ & $c_{l_{j^{*}}^{*}, q^{*}}^{*}$ & $c_{l_{j^{*}}^{*}, p u^{*}}^{*}$ \\
$\vdots$ & $\vdots$ & $\cdots$ & $\vdots$ & $\cdots$ & $\vdots$ & $\vdots$ \\
$l_{n^{*}}^{*}$ & $c_{l_{n^{*}, u_{1}}^{*}}^{*}$ & $\cdots$ & $c_{l_{n^{*}}^{*}, u_{f}}^{*}$ & $c_{l^{*}, R^{*}}^{*}$ & $c_{l_{n^{*}}^{*}, q^{*}}^{*}$ & $c_{l_{n^{*}}^{*}, p u^{*}}^{*}$ \\
$Q^{*}$ & $c_{Q^{*}, u_{1}}^{*}$ & $\cdots$ & $c_{Q^{*}, u_{f}}^{*}$ & $c_{Q^{*}, R^{*}}^{*}$ & $c_{Q^{*}, q^{*}}^{*}$ & $c_{Q^{*}, p u^{*}}^{*}$ \\
$p l^{*}$ & $c_{p l^{*}, u_{1}}^{*}$ & $\cdots$ & $c_{p l^{*}, u_{f}}^{*}$ & $c_{p l^{*}, R^{*}}^{*}$ & $c_{p l^{*}, q^{*}}^{*}$ & $c_{p l^{*}, p u^{*}}^{*}$ \\
$p u_{1}^{*}$ & $c_{p u_{1}^{*}, u_{1}}^{*}$ & $\cdots$ & $c_{p u_{1}^{*}, u_{f}}^{*}$ & $c_{p u_{1}^{*}, R^{*}}^{*}$ & $c_{p u_{1}^{*}, q^{*}}^{*}$ & $c_{p u_{1}^{*}, p u^{*}}^{*}$ \\
\hline
\end{tabular}


where $\quad L^{*}=\left\{l_{1}^{*}, \ldots, l_{j^{*}}^{*}, \ldots, l_{n^{*}}^{*}, Q^{*}, p l^{*}, p u_{1}^{*}\right\}$, $U \quad=\quad\left\{u_{1}, \ldots, u_{g}, \ldots, u_{f}, R^{*}, q^{*}, p u^{*}\right\}$ and $L^{*} \quad \subset$ $L$ and for $1 \leq j^{*} \leq n^{*}, \quad 1 \leq g \leq f$, $\left\{c_{l_{j^{*}}^{*}, u_{g}}^{*}, c_{l_{j^{*}}^{*}, R^{*}}, c_{l_{j^{*}}^{*}, q^{*}}^{*}, c_{l_{j^{*}}^{*}, p u^{*}}^{*}, c_{p u_{1}^{*}, q^{*}}^{*}, c_{p u_{1}^{*}, u_{g}}^{*}, c_{p u_{1}^{*}, R}^{*}, c_{Q^{*}, u_{f}}^{*}\right.$, $\left.c_{p l^{*}, u_{f}}^{*}\right\}$ and $c_{p u_{1}^{*}, p u^{*}}^{*}$ are IFPs, having meaning as defined in the generalized 2-S IFTP.

We also define the IFIM

$$
X\left[L^{J}, U\right]=\begin{array}{c|ccccc} 
& u_{1} & \ldots & u_{g} & \ldots & u_{f} \\
\hline l_{1^{*}}^{*} & x_{l^{*}}^{*}, u_{1} & \cdots & x_{l_{1^{*}}^{*}, u_{g}} & \cdots & x_{l_{1^{*}}^{*}, u_{f}} \\
\vdots & \vdots & \ddots & \vdots & \ddots & \vdots \\
l_{n^{*}}^{*} & x_{l_{n^{*}}^{*}, u_{1}} & \ldots & x_{l_{n^{*}}^{*}, u_{g}} & \ldots & x_{l_{n^{*}}^{*}, u_{f}}
\end{array}
$$

where $L^{J}=\left\{l_{1^{*}}^{*}, l_{2^{*}}^{*}, \ldots, l_{n^{*}}^{*}\right\}, U=\left\{u_{1}, u_{2}, \ldots, u_{f}\right\}$, and for $1 \leq$ $j^{*} \leq n^{*}, 1 \leq g \leq f: x_{l_{j^{*}}^{*}, u_{g}}=\left\langle\rho_{l_{j^{*}}^{*}, u_{g}}, \sigma_{l_{j^{*}}^{*}, u_{g}}\right\rangle$ are the number of units of the product, transported from the $l_{j^{*}}^{*}$-th reseller to $u_{g}$-th destination.

Go to Step 5.

Step 5. Let construct IFIM matrix:

$$
\left.C_{1}=\operatorname{pr}_{R S, R^{*}}\left(\alpha_{K^{I}, \#_{q}}\left(X_{o p t}, R^{*}\right)^{T}\right)\right) .
$$

Then $C^{*}:=C^{*} \oplus_{(\circ, *)} C_{1}$.

So, the quantities of product purchased in this way by the resellers from the set $R S$ are set in the column $R^{*}$ of the matrix $C^{*}$. Also, the elements $\left\{c_{l_{j^{*}}^{*}, q^{*}}^{*}, c_{Q^{*}, u_{g}}^{*}, c_{p l^{*}, u_{g}}^{*}\right\}$ (for $\left.1 \leq j^{*} \leq n^{*}, 1 \leq g \leq f\right)$ are introduced in $C^{*}$.

Construct the matrix $E\left[K /\left\{Q, p l, p u_{1}\right\}, L /\{R, p u\}\right]$

$$
=C_{\left(\left\{Q, p l, p u_{1}\right\},\{R, p u\}\right)} \otimes_{(\min , \max )} X_{o p t} .
$$

Go to Step 6.

Step 6. Through the following operations we will find the average price of the $l_{j^{*}}^{*}$-th reseller $\in R S$ to purchase a single quantity of product.

Then construct the IM $C_{2 a}=\alpha_{K^{I}, \#_{2}}\left(E, p u^{*}\right)^{T}$;

For $1 \leq j^{*} \leq n^{*}$ do:

$\{$ Construct the matrices:

$$
C_{2 b}\left[l_{j^{*}}^{*}, R_{j^{*}}^{*},\left\{C_{2 a_{l_{j^{*}}^{*}}, p u^{*}} / C_{l_{j^{*}}^{*}, R_{j^{*}}^{*}}^{*}\right\}\right],
$$

in which we use the operation division of IFPs (2):

$$
\left.C^{*}:=C^{*} \oplus_{(\circ, *)}\left[\perp ; \frac{p u^{*}}{R^{*}}\right] C_{2 b} \cdot\right\}
$$

Go to Step 7.

Step 7. The following operations will reflect in the column $p u^{*}$ of the matrix $C^{*}$ the final selling prices of a unit quantity of the product together with its surplus charge above the purchase price.

Let us construct the matrices

$$
C_{3}=\left[\perp ; \frac{p u^{*}}{q^{*}}\right]\left\{p r_{R S, q^{*}} C^{*}\right\}
$$

and $C_{4}=p r_{R S, p u^{*}} C^{*}$.

Let us perform operation $C^{*}:=C^{*} \oplus_{(\circ, *)} C_{3} \otimes C_{4}$.

Go to Step 8.
Step 8. Through the following operations, the elements $c_{l^{*} j^{*}, u_{g}}^{*}$ (for $1 \leq g \leq f$ ) of the matrix $C^{*}$ will contain the final selling price per unit of product, including the unit price and its transportation price from the $l^{*} j^{*}$-th reseller to $u_{g}$-th destination.

For $1 \leq j^{*} \leq n^{*}, 1 \leq g \leq f$, do following:

$$
\begin{gathered}
\left\{C_{l^{*} j^{*}, u_{g}}^{*}=\left\{p r_{l_{j^{*}}^{*}, u_{g}} C^{*}\right\} \oplus_{(\circ, *)}\left[\perp ; \frac{u_{g}}{p u^{*}}\right]\left\{p r_{l_{j^{*}}^{*}, p u^{*}} C^{*}\right\} ;\right. \\
\left.C^{*}:=C^{*} \oplus_{(\circ, *)} C_{l_{j^{*}}^{*}, u_{g}}^{*}\right\}
\end{gathered}
$$

Go to Step 9.

Step 9. Determining the optimal plan at second stage of the 2-S IFTP - $X^{*}\left[L^{J}, U,\left\{x_{l^{*}}^{*}, u_{g}\right\}\right]$ after execution of one of the algorithms, presented in [47], [48], [52] with the obtained cost IFIM $C^{*}$. The optimal intuitionistic fuzzy transportation cost at the second stage is calculated by:

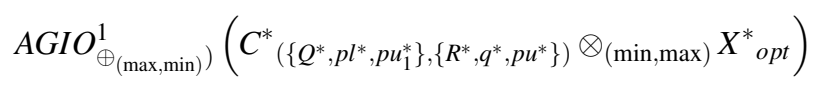

or $A G I O_{\oplus_{\left(\wedge_{2}\right)}}^{2}\left(C^{*}{ }_{\left(\left\{Q^{*}, p l^{*}, p u_{1}^{*}\right\},\left\{R^{*}, q^{*}, p u^{*}\right\}\right)} \otimes_{\left(\vee_{2}\right)} X^{*}\right.$ opt $)$, where $\vee_{2}$ and $\wedge_{2}$ are the operations from (1).

Step 10. The optimal intuitionistic fuzzy transportation cost for the problem is calculated by:

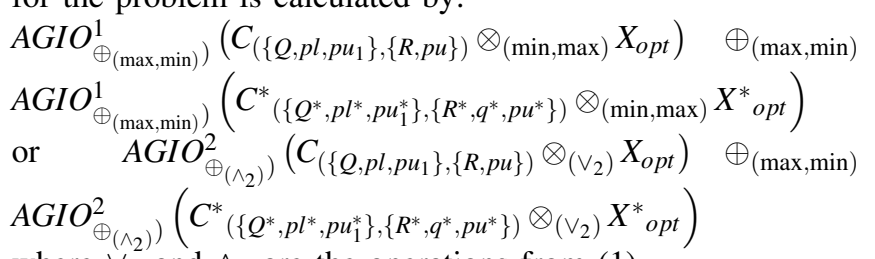
where $\vee_{2}$ and $\wedge_{2}$ are the operations from (1).

\section{An Application of the Algorithm for Solution OF 2-S IFTP}

In this section we will define 2-S IFTP extending the IFTP from [48]: A trader supplies a product to 4 different companies $\left\{l_{1}, l_{2}, l_{3}, l_{4}\right\}$. Let a product be produced at the manufacturers $\left\{k_{1}, k_{2}, k_{3}\right\}$ in quantities $c_{k_{i}, R}$ (for $1 \leq i \leq 3$ ). Let the companies $\left(\left\{l_{1}, l_{2}, l_{3}, l_{4}\right\}\right)$ demand this product in an quantity of $c_{Q, l_{j}}$ (for $1 \leq j \leq 4$ ) and $c_{p l, l_{j}}$ (for $1 \leq j \leq 4$ ) are intuitionistic fuzzy limits to the transportation costs of delivery a particular product from the $k_{i}$-th source to the $l_{j}$-th destination. Let some of the buyers $R S=\left\{l_{1}, l_{2}, l_{3}\right\}(R S \subset L)$ become resellers. The resellers $\left\{l_{1}, l_{2}, l_{3}\right\}$ want to sell quantities of the product not only purchased, but also from own production or stocks at a surplus charge $c_{l_{j^{*}}^{*}, q^{*}}^{*}$ (for $1 \leq j^{*} \leq 3$ ) for an product unit to other consumers $\left\{u_{1}, u_{2}, u_{3}, u_{4}\right\}$, in quantities $c_{l_{j^{*}}^{*}, R^{*}}^{*}$ (for $1 \leq$ $\left.j^{*} \leq 3\right)$. Consumers need this product in an amount of $c_{Q^{*}, u_{g}}^{*}$ (for $1 \leq g \leq 4$ ). Let $c_{l^{*}}^{*}, u_{g}$ (for $1 \leq j^{*} \leq n^{*}, 1 \leq g \leq f$ ) be the total cost for the purchase of one unit quantity of the product from the $l_{j^{*}}^{*}$-th reseller to $u_{g}$-th destination; $x_{l^{*}}^{*}, u_{g}-$ the number of units of the product, transported from the $l_{j^{*}}^{*}$-th reseller to $u_{g}$-th destination; $c_{l_{j^{*}}^{*}, p u^{*}}^{*},\left(\right.$ for $\left.1 \leq j^{*} \leq 3\right)-$ is the price of a product unit of the $l_{j^{*}}^{*}$ th reseller; $c_{p l^{*}, u_{g}}^{*},($ for $1 \leq g \leq 4)-$ 
upper limit of the price at which the $u_{g}$-th consumer wish to purchase the product.

The purpose of the 2-S IFTP is to meet the requests of all users $\left\{l_{1}, \ldots, l_{4}\right\}$ and $\left\{u_{1}, u_{2}, u_{3}\right\}$ so that the intuitionistic fuzzy transportation cost is minimum.

All elements of the transportation problem are intuitionistic fuzzy due to several uncertainties.

Let us apply the proposed approach in the Sect. III.

1) Solution of the First Stage of the 2-S IFTP: Step 1. At starting of the algorithm for solution of the problem, the cost IM $C$ is created. $c_{k_{i}, l_{j}}$ (for $1 \leq i \leq 3,1 \leq j \leq 4$ ) is the IF cost for transporting a unit quantity of the product from the $k_{i}$-th producer to the $l_{j}$-th user.

$$
C[K, L]=\left\{\begin{array}{c|cccc} 
& l_{1} & l_{2} & l_{3} & \ldots \\
\hline k_{1} & \langle 0.6,0.2\rangle & \langle 0.7,0.1\rangle & \langle 0.3,0.1\rangle & \ldots \\
k_{2} & \langle 0.5,0.3\rangle & \langle 0.4,0.1\rangle & \langle 0.5,0.1\rangle & \ldots \\
k_{3} & \langle 0.4,0.2\rangle & \langle 0.3,0.2\rangle & \langle 0.6,0.1\rangle & \ldots \\
Q & \langle 0.4,0.2\rangle & \langle 0.5,0.3\rangle & \langle 0.6,0.2\rangle & \ldots \\
p l & \langle 0.65,0.3\rangle & \langle 0.6,0.4\rangle & \langle 0.75,0.1\rangle & \ldots \\
p u_{1} & \langle\perp, \perp\rangle & \langle\perp, \perp\rangle & \langle\perp, \perp\rangle & \ldots \\
\ldots & l_{4} & R & p u & \\
\ldots & \ldots 0.8,0.1\rangle & \langle 0.5,0.2\rangle & \langle\perp, \perp\rangle \\
& \ldots & \langle 0.3,0.2\rangle & \langle 0.7,0.1\rangle & \langle\perp, \perp\rangle \\
& \ldots & \langle 0.7,0.2\rangle & \langle 0.4,0.5\rangle & \langle\perp, \perp\rangle \\
\\
\ldots & \langle 0.06,0.02\rangle & \langle\perp, \perp\rangle & \langle\perp, \perp\rangle & \\
& \ldots & \langle 0.75,0.1\rangle & \langle\perp, \perp\rangle & \langle\perp, \perp\rangle \\
\end{array}\right.
$$

Let $x_{k_{i}, l_{j}}$ is the number of units of the product, transported from the $k_{i}$-th producer to $l_{j}$-th destination (for $1 \leq i \leq 3$ and $1 \leq j \leq 4)$ and is an element of IFIM $X$ with initial elements $\langle\perp, \perp\rangle$. The trader wants to satisfy the required quantities of the users so that the intuitionistic fuzzy transportation cost is minimum

Step 2. The conditions for limiting the transportation costs are checked according to proposed approach in [48]. The problem is also balanced.

The IM $C$ is transformed in this form following the IF algorithm in [48]:

$$
C[K, L]=\left\{\begin{array}{c|cccc} 
& l_{1} & l_{2} & l_{3} & \ldots \\
\hline k_{1} & \langle 0.6,0.2\rangle & \langle 1,0\rangle & \langle 0.3,0.1\rangle & \ldots \\
k_{2} & \langle 0.5,0.3\rangle & \langle 0.4,0.1\rangle & \langle 0.5,0.1\rangle & \ldots \\
k_{3} & \langle 0.4,0.2\rangle & \langle 0.3,0.2\rangle & \langle 0.6,0.1\rangle & \ldots \\
Q & \langle 0.4,0.2\rangle & \langle 0.5,0.3\rangle & \langle 0.6,0.2\rangle & \ldots \\
p l & \langle 0.65,0.3\rangle & \langle 0.6,0.4\rangle & \langle 0.75,0.1\rangle & \ldots \\
p u_{1} & \langle\perp, \perp\rangle & \langle\perp, \perp\rangle & \langle\perp, \perp\rangle & \ldots \\
\ldots & l_{4} & R & p u & \\
\ldots & \langle 1,0\rangle & \langle 0.5,0.2\rangle & \langle\perp, \perp\rangle \\
\ldots & \langle 0.3,0.2\rangle & \langle 0.7,0.1\rangle & \langle\perp, \perp\rangle & \\
\ldots & \langle 0.7,0.2\rangle & \langle 0.4,0.5\rangle & \langle\perp, \perp\rangle & \\
\ldots & \langle 0.06,0.02\rangle & \langle\perp, \perp\rangle & \langle\perp, \perp\rangle & \\
\ldots & \langle 0.65,0.3\rangle & \langle\perp, \perp\rangle & \langle\perp, \perp\rangle & \\
\ldots & \langle\perp, \perp\rangle & \langle\perp, \perp\rangle & \langle\perp, \perp\rangle
\end{array}\right.
$$

For solving the first stage we can apply the zero-point algorithm for IFTP with IFIMs $C$ and $X$, outlined in [48].
Step 3. The intuitionistic fuzzy optimal solution, presented by the IM $X_{o p t}$ is non-degenerated, it includes 6 occupied cells. The IM $X_{o p t}$ has the following form:

$X_{o p t}=\left\{\begin{array}{c|cccc} & l_{1} & l_{2} & l_{3} & l_{4} \\ \hline k_{1} & \langle 0,1\rangle & \langle 0,1\rangle & \langle 0.5,0.2\rangle & \langle 0,1\rangle \\ k_{2} & \langle 0.4,0.2\rangle & \langle 0.1,0.8\rangle & \langle 0.1,0.4\rangle & \langle 0.06,0.02\rangle \\ k_{3} & \langle 0,1\rangle & \langle 0.4,0.5\rangle & \langle 0,1\rangle & \langle 0,1\rangle\end{array}\right.$.

The optimal intuitionistic fuzzy optimal solution $X_{o p t}\left[K *, L *,\left\{x_{k_{i}, l_{j}}\right\}\right]$ is obtained. The optimal intuitionistic fuzzy transportation cost is:

$$
\begin{aligned}
& A G I O_{\left.\oplus_{(\max , \min )}\right)}^{1}\left(C_{\left(\left\{Q, p l, p u_{1}\right\},\{R, p u\}\right)} \otimes_{(\min , \max )} X_{o p t}\right)=\langle 0.4,0.2\rangle \\
& \text { or } \\
& { }^{A G I O_{\oplus_{\left(\wedge_{2}\right)}}^{2}}\left(C_{\left(\left\{Q, p l, p u_{1}\right\},\{R, p u\}\right)} \otimes_{\left(\vee_{2}\right)} X_{o p t}\right)=\langle 0.464,0.006\rangle .
\end{aligned}
$$

The degree of membership (acceptance) of this optimal solution is equal to 0.4 (or 0.464) and its degree of nonmembership (non-acceptance) is equal to 0.2 (or 0.006).

The ranking function $R$, defined in (3), we can use to rank alternatives of decision-making process. For the obtained optimal solution by IFZPM, the distance between the optimal solution to the pair $\langle 1,0\rangle$ is equal to $R_{\langle 0.4 ; 0.2\rangle}=0.42$ $\left(R_{\langle 0.464 ; 0.006\rangle}=0.41\right)$.

2) Solution of the Second Stage of the 2-S IFTP: To find the optimal solution for the second stage, we propose the following algorithm, described by program code, which is a part of Microsoft Visual Studio.NET 2010 C project.

Step 4. The following cost IFIM $C^{*}\left[L^{*}, U\right]$ is created:

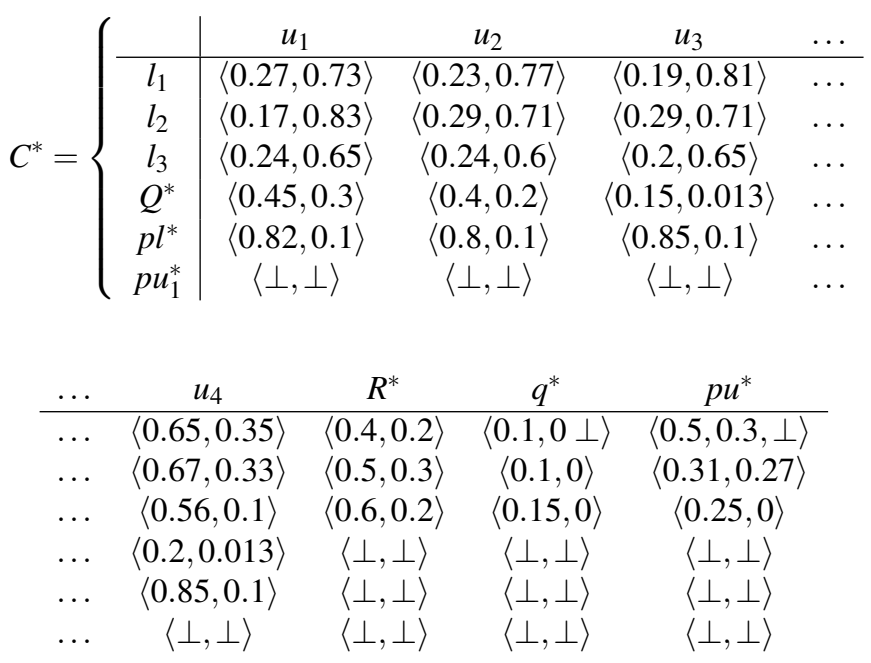

where $\quad L^{*}=\left\{l_{1}, \ldots, l_{3}, Q^{*}, p l^{*}, p u_{1}^{*}\right\}, \quad U=$ $\left\{u_{1}, \ldots, u_{4}, R^{*}, q^{*}, p u^{*}\right\}$ and all elements are IFPs. The quantities of product purchased on the first stage by the resellers from the set $R S$ are set in the column $R^{*}$ of the matrix $C^{*}$. 
We also define

$$
X\left[L^{J}, U\right]=\left\{\begin{array}{c|cccc} 
& u_{1} & u_{2} & u_{3} & u_{4} \\
\hline l_{1} & \langle 0,1\rangle & \langle 0,1\rangle & \langle 0,1\rangle & \langle 0,1\rangle \\
l_{2} & \langle 0,1\rangle & \langle 0,1\rangle & \langle 0,1\rangle & \langle 0,1\rangle \\
l_{3} & \langle 0,1\rangle & \langle 0,1\rangle & \langle 0,1\rangle & \langle 0,1\rangle
\end{array}\right\},
$$

$L^{J}=\left\{l_{1}, l_{2}, l_{3}\right\}, \quad U=\left\{u_{1}, u_{2}, u_{3}, u_{4}\right\}$ and for $1 \leq j^{*} \leq 3$, $1 \leq g \leq 4: x_{j^{*}, u_{g}}=\left\langle\rho_{l_{j^{*}}^{*}, u_{g}}, \sigma_{l_{j^{*}}^{*}, u_{g}}\right\rangle$ are the number of units of the product, transported from the $l_{j^{*}}^{*}$-th reseller to $u_{g}$-th destination.

Step 5. The average prices of the resellers $l_{1}, l_{2}, l_{3}$ to purchase a single quantity of product are calculated. The IFIM $C^{*}\left[L^{*}, U\right]$ is changed as follows:

$\left\{\begin{array}{c|cccc} & \ldots & R^{*} & q^{*} & p u^{*} \\ \hline l_{1} & \ldots & \langle 0.4,0.2\rangle & \langle\perp, \perp\rangle & \langle 0.5,0.3\rangle \\ l_{2} & \ldots & \langle 0.5,0.3\rangle & \langle\perp, \perp\rangle & \langle 0.31,0.27\rangle \\ l_{3} & \ldots & \langle 0.6,0.2\rangle & \langle\perp, \perp\rangle & \langle 0.25,0\rangle \\ Q^{*} & \ldots & \langle\perp, \perp\rangle & \langle\perp, \perp\rangle & \langle\perp, \perp\rangle \\ p l^{*} & \ldots & \langle\perp, \perp\rangle & \langle\perp, \perp\rangle & \langle\perp, \perp\rangle \\ p u_{1}^{*} & \ldots & \langle\perp, \perp\rangle & \langle\perp, \perp\rangle & \langle\perp, \perp\rangle\end{array}\right\}$

Step 6. The column $p u^{*}$ of the matrix $C^{*}$ contains the final selling prices of a unit quantity of the product together with its mark-up above the purchase price. The elements $c_{l^{*} j^{*}, u_{g}}^{*}$ (for $1 \leq j^{*} \leq 3,1 \leq g \leq f$ ) of the matrix $C^{*}$ contain the final selling price per unit of product, including the unit price and its transportation price from the $l^{*} j^{*}$-th reseller to $u_{g}$-th destination. $C^{*}$ obtains the following form:

$C^{*}=\left\{\begin{array}{c|cccc} & u_{1} & u_{2} & u_{3} & \ldots \\ \hline l_{1} & \langle 0.32,0.55\rangle & \langle 0.28,0.55\rangle & \langle 0.24,0.7\rangle & \ldots \\ l_{2} & \langle 0.2,0.7\rangle & \langle 0.32,0.55\rangle & \langle 0.32,0.6\rangle & \ldots \\ l_{3} & \langle 0.28,0.65\rangle & \langle 0.28,0.6\rangle & \langle 0.2,0.65\rangle & \ldots \\ Q^{*} & \langle 0.45,0.3\rangle & \langle 0.4,0.2\rangle & \langle 0.15,0.013\rangle & \ldots \\ p l^{*} & \langle 0.82,0.1\rangle & \langle 0.8,0.1\rangle & \langle 0.85,0.1\rangle & \ldots \\ p u_{1}^{*} & \langle\perp, \perp\rangle & \langle\perp, \perp\rangle & \langle\perp, \perp\rangle & \ldots\end{array}\right.$

\begin{tabular}{ccccc}
$\ldots$ & $u_{4}$ & $R^{*}$ & $q^{*}$ & $p u^{*}$ \\
\hline$\ldots$ & $\langle 0.7,0.1\rangle$ & $\langle 0.4,0.2\rangle$ & $\langle 0.1,0 \perp\rangle$ & $\langle 0.05,0.3, \perp\rangle$ \\
$\ldots$ & $\langle 0.7,0.1\rangle$ & $\langle 0.5,0.3\rangle$ & $\langle 0.1,0\rangle$ & $\langle 0.03,0.27\rangle$ \\
$\ldots$ & $\langle 0.6,0.1\rangle$ & $\langle 0.6,0.2\rangle$ & $\langle 0.15,0\rangle$ & $\langle 0.038,0\rangle$ \\
$\ldots$ & $\langle 0.2,0.013\rangle$ & $\langle\perp, \perp\rangle$ & $\langle\perp, \perp\rangle$ & $\langle\perp, \perp\rangle$ \\
$\ldots$ & $\langle 0.85,0.1\rangle$ & $\langle\perp, \perp\rangle$ & $\langle\perp, \perp\rangle$ & $\langle\perp, \perp\rangle$ \\
$\ldots$ & $\langle\perp, \perp\rangle$ & $\langle\perp, \perp\rangle$ & $\langle\perp, \perp\rangle$ & $\langle\perp, \perp\rangle$
\end{tabular}

Step 7. The problem is balanced. Then the requirements for an upper limit on the price at which consumers have the opportunity to purchase the necessary quantities of the product are checked. After execution of the algorithm, presented in [48], with the obtained cost IFIMs $C^{*}$ and $X^{*}$, we obtain the following optimal plan $X^{*}\left[L^{J}, U,\left\{x_{l_{j^{*}}^{*}, u_{g}}^{*}\right\}\right]$ for the second stage of the problem:

$\left\{\begin{array}{c|cccc} & u_{1} & u_{2} & u_{3} & u_{4} \\ \hline l_{1} & \langle 0,1\rangle & \langle 0.35,0.65\rangle & \langle 0,1\rangle & \langle 0,1\rangle \\ l_{2} & \langle 0.45,0.3\rangle & \langle 0.05,0.6\rangle & \langle 0,1\rangle & \langle 0,1\rangle \\ l_{3} & \langle 0,1\rangle & \langle 0.15,0.23\rangle & \langle 0.15,0.013\rangle & \langle 0.2,0.013\rangle\end{array}\right\}$

The intuitionistic fuzzy optimal solution, presented by the IM $X_{o p t}^{*}$ is non-degenerated, it includes 6 occupied cells. The optimal intuitionistic fuzzy transportation cost at the second stage is calculated by:

$$
\begin{gathered}
\begin{array}{c}
\operatorname{AGIO}_{\left.\oplus_{(\max , \min )}\right)}^{1}\left(C^{*}{ }_{\left(\left\{Q^{*}, p l^{*}, p u_{1}^{*}\right\},\left\{R^{*}, q^{*}, p u^{*}\right\}\right)} \otimes_{(\min , \max )} X^{*}{ }_{\text {opt }}\right) \\
=\langle 0.28,0.1\rangle
\end{array} \\
\text { or } \begin{array}{c}
\operatorname{AGIO}_{\oplus_{\left(\wedge_{2}\right)}}^{2}\left(C^{*}\left(\left\{Q^{*}, p l^{*}, p u_{1}^{*}\right\},\left\{R^{*}, q^{*}, p u^{*}\right\}\right)\right. \\
\left.\otimes_{\left(\vee_{2}\right)} X^{*} \text { opt }\right) \\
=\langle 0.31,0.04\rangle,
\end{array}
\end{gathered}
$$

where $\vee_{2}$ and $\wedge_{2}$ are the operations from (1). The degree of membership (acceptance) of this optimal solution is equal to 0.28 (or 0.31 ) and the its degree of non-membership (nonacceptance) is equal to 0.1 (or 0.04). For the obtained optimal solution by IFZPM, the distance between the optimal solution to the pair $\langle 1,0\rangle$ is equal to $R_{\langle 0.28 ; 0.1\rangle}=0.58\left(R_{\langle 0.31 ; 0.04\rangle}=\right.$ $0.57)$.

Step 8. The optimal intuitionistic fuzzy transportation cost for the problem is calculated by: $\langle 0.4,0.2\rangle \oplus_{(\text {max.min })}$ $\langle 0.28,0.1\rangle($ or $\langle 0.31,0.4\rangle)=\langle 0.4,0.1\rangle($ or $\langle 0.4,0.4\rangle)$.

The degree of membership (acceptance) of this optimal solution is equal to 0.4 .

The example illustrates the reliability of the proposed algorithm in Section III to the studied 2-S IFTP.

\section{CONCLUSION}

The apparatus of IMs provides the ability to expand the existing transportation problems to formulate non-existent ones to find strategic decisions for logistics management in uncertain environment. It is proposed for the first time, extending the approach in [48], to model and find the optimal solution of a 2-S IFTP using the concepts of the IMs and IFSs. The formulated IFTP has additional constraints: upper limits to the transportation costs and a surplus charge on reseller sales prices. The proposed algorithm for solution of the 2-S IFTP is illustrated with a numerical example. The advantages of the proposed algorithm are that it can be easy generalized to the multidimensional intuitionistic fuzzy TPs [23] and also can be applied to both the TP with crisp parameters and with intuitionistic fuzzy ones. In the future, we will extend the proposed approach to the interval-valued intuitionistic fuzzy TPs [25] and will apply it over real life TPs.

\section{REFERENCES}

[1] A. Edwuard Samuel, "Improved zero point method," Applied mathematical sciences, vol. 6 (109), 2012, pp. 5421-5426.

[2] A. Gani, A. Samuel, D. Anuradha, "Simplex type algorithm for solving fuzzy transportation problem," Tamsui Oxf. J. Inf. Math. Sci., vol. 27, 2011, pp. 89-98.

[3] A. Gani, K. Razak, "Two stage fuzzy transportation problem," J Phys Sci, vol. 10, 2006, pp. 63-69.

[4] A. Gani, S. Abbas, "A new average method for solving intuitionistic fuzzy transportation problem," International Journal of Pure and Applied Mathematics, vol. 93 (4), 2014, pp. 491-499.

,[5] A. Kaur, A. Kumar, "A new approach for solving fuzzy transportation problems using generalized trapezoidal fuzzy numbers," Applied Soft Computing, vol. 12 (3), 2012, pp. 1201-1213.

[6] A. Kaur, J. Kacprzyk and A. Kumar, Fuzzy transportation and transshipment problems, Studies in fuziness and soft computing, vol. 385, 2020. 
[7] A. Patil, S. Chandgude, "Fuzzy Hungarian Approach for Transportation Model," International Journal of Mechanical and Industrial Engineering, vol. 2 (1), pp. 77-80, 2012.

[8] A.E. Samuel, M. Venkatachalapathy, "Modified vogel's approximation method for fuzzy transportation problems," Appl. Math. Sci., vol. 5, 2011, pp. 1367-1372.

[9] B. Atanassov, Quantitative methods in business management, Publ. houseTedIna, Varna; 1994. (in Bulgarian)

[10] B. Riecan, A. Atanassov, "Operation division by $n$ over intuitionistic fuzzy sets," NIFS, vol. 16, No. 4, 2010, pp. 1-4.

[11] S. Dinagar, K. Palanivel, "The transportation problem in fuzzy environment," Int. J. Algorithms Comput. Math., vol. 2, 2009, pp. 65-71.

[12] E. Szmidt, J. Kacprzyk, "Amount of information and its reliability in the ranking of Atanassov's intuitionistic fuzzy alternatives," in: RakusAndersson, E., Yager, R., Ichalkaranje, N., Jain, L.C. (eds.), Recent Advances in Decision Making, SCI, Springer, Heidelberg, vol. 222 2009, pp. 7-19.

[13] F. Jimenez, J. Verdegay, "Solving fuzzy solid transportation problems by an evolutionary algorithm based parametric approach," European Journal of Operational Research, vol. 117 (3),1999, pp. 485-510.

[14] F. Hitchcock, "The distribution of a product from several sources to numerous localities," Journal of Mathematical Physics, vol. 20, 1941, pp. 224-230.

[15] G. Gupta, A. Kumar, M. Sharma, "A Note on A New Method for Solving Fuzzy Linear Programming Problems Based on the Fuzzy Linear Complementary Problem (FLCP)," International Journal of Fuzzy Systems, 2016, pp. 1-5.

[16] H. Arsham, A. Khan, "A simplex type algorithm for general transportation problems-An alternative to stepping stone," Journal of Operational Research Society, vol. 40 (6), 2017, pp. 581-590.

[17] H. Basirzadeh, "An approach for solving fuzzy transportation problem, " Appl. Math. Sci., vol. 5, 2011, pp. 1549-1566.

[18] K. Atanassov, "Intuitionistic Fuzzy Sets," VII ITKR Session, Sofia 20-23 June 1983 (Deposed in Centr. Sci.-Techn. Library of the Bulg. Acad. of Sci., 1697/84) (in Bulgarian). Reprinted: Int. J. Bioautomation, vol. 20(S1), 2016, pp. S1-S6.

[19] K. Atanassov, "Generalized index matrices," Comptes rendus de l'Academie Bulgare des Sciences, vol. 40(11), 1987, pp. 15-18.

[20] K. Atanassov, On Intuitionistic Fuzzy Sets Theory, STUDFUZZ. Springer, Heidelberg, vol. 283; DOI:10.1007/978-3-642-29127-2, 2012.

[21] K. Atanassov, Index Matrices: Towards an Augmented Matrix Calculus. Studies in Computational Intelligence, Springer, Cham, vol. 573; DOI 10.1007/978-3-319-10945-9, 2014.

[22] K. Atanassov, "Intuitionistic Fuzzy Logics," Studies in Fuzziness and Soft Computing, Springer, vol. 351, DOI:10.1007/978-3-319-48953-7, 2017.

[23] K. Atanassov, "n-Dimensional extended index matrices Part 1," Advanced Studies in Contemporary Mathematics, vol. 28 (2), 2018, pp. 245-259.

[24] K. Atanassov, "Remark on an intuitionistic fuzzy operation division," Annual of Informatics Section, Union of Scientists in Bulgaria, vol. 10, 2019 (in press)

[25] K. Atanassov, G. Gargov, "Interval valued intuitionistic fuzzy sets," Fuzzy sets and systems, vol. 31 (3), 1989, pp. 343-349.

[26] K. Atanassov, E. Szmidt, J. Kacprzyk, "On intuitionistic fuzzy pairs," Notes on Intuitionistic Fuzzy Sets, vol. 19 (3), 2013, pp. 1-13.

[27] K. Kathirvel, K. Balamurugan, "Method for solving fuzzy transportation problem using trapezoidal fuzzy numbers," International Journal of Engineering Research and Applications, vol. 2 (5), 2012, pp. 2154-2158.

[28] K. Kathirvel, K. Balamurugan, "Method for solving unbalanced transportation problems using trapezoidal fuzzy numbers," International Journal of Engineering Research and Applications, vol. 3 (4), 2013, pp. 2591-2596.

[29] L. Zadeh, Fuzzy Sets, Information and Control, vol. 8 (3), 338-353; 1965.

[30] M. Purushothkumar, M. Ananthanarayanan, S. Dhanasekar, "Fuzzy zero suffix Algorithm to solve Fully Fuzzy Transportation Problems,' International Journal of Pure and Applied Mathematics, vol. 119 (9), 2018, pp. 79-88

[31] M. Shanmugasundari, K. Ganesan, "A novel approach for the fuzzy optimal solution of fuzzy transportation problem," International journal of Engineering research and applications, vol. 3 (1), 2013, pp. 14161424

[32] P. Jayaraman, R. Jahirhussain, "Fuzzy optimal transportation problem by improved zero suffix method via Robust Ranking technique," International Journal of Fuzzy Mathematics and systems, vol. 3 (4), 2013 pp. 303-311.

[33] P. Kaur, K. Dahiya, "Two-stage interval time minimization transportation problem with capacity constraints," Innov Syst Des Eng, vol. 6, 2015 pp.79-85.

[34] P. Pandian, G. Natarajan, "A new algorithm for finding a fuzzy optimal solution for fuzzy transportation problems," Applied Mathematical Sciences, vol. 4, 2010, pp. 79- 90.

[35] R. Antony, S. Savarimuthu, T. Pathinathan, "Method for solving the transportation problem using triangular intuitionistic fuzzy number,' International Journal of Computing Algorithm, vol. 03, 2014, pp. 590605.

[36] R. Jahirhussain, P. Jayaraman, "Fuzzy optimal transportation problem by improved zero suffix method via robust rank techniques," International Journal of Fuzzy Mathematics and Systems (IJFMS), vol. 3, 2013, pp. 303-311.

[37] R. Jahihussain , P. Jayaraman, "A new method for obtaining an optinal solution for fuzzy transportation problems," International Journal of Mathematical Archive, vol. 4 (11), 2013, pp. 256-263.

[38] S. K. Bharati, R. Malhotra, "Two stage intuitionistic fuzzy time minimizing TP based on generalized Zadeh's extension principle," Int J Syst Assur Eng Manag, vol. 8, 2017, pp. 1142-1449. DOI: 10.1007/s13198 017-0613-9

[39] S. Chanas, W. Kolodziejckzy, A. Machaj, "A fuzzy approach to the transportation problem," Fuzzy Sets and Systems, vol. 13, 1984, pp. 211-221.

[40] S. Dhanasekar, S. Hariharan, P. Sekar, "Fuzzy Hungarian MODI Algorithm to solve fully fuzzy transportation problems," Int. J. Fuzzy Syst., vol. 19 (5), 2017, pp. 1479-1491.

[41] S. Liu, C. Kao, "Solving fuzzy transportation problems based on extension principle," Eur. J. Oper. Res., vol. 153, 2004, pp. 661-674.

[42] S. Midya, SS. K. Roy, V. F. Yu, "Intuitionistic fuzzy multi-stage multiobjective fixed-charge solid transportation problem in a green supply chain,” Int. J. Mach. Learn. \& Cyber., vol. 12, 2021, pp. 699-717.

[43] S. Malhotra, R. Malhotra, "A polynomial Algorithm for a Two - Stage Time Minimizing Transportation Problem, OPSEARCH, vol. 39, 2002, pp. 251-266.

[44] V. Sudhagar, V. Navaneethakumar, "Solving the Multiobjective two stage fuzzy transportation problem by zero suffix method," Journal of Mathematics Research, vol. 2 (4), 2010, pp. 135-140.

[45] V. Traneva, "Internal operations over 3-dimensional extended index matrices," Proceedings of the Jangieon Mathematical Society, vol. 18 (4), 2015, pp. 547-569.

[46] V. Traneva, "One application of the index matrices for a solution of a transportation problem," Advanced Studies in Contemporary Mathematics, vol. 26 (4), 2016, pp. 703-715.

[47] V. Traneva, P. Marinov, K. Atanassov, "Index matrix interpretations of a new transportation-type problem," Comptes rendus de l'Academie Bulgare des Sciences, vol. 69 (10), 2016, pp. 1275-1283.

[48] V. Traneva, S. Tranev, "Intuitionistic Fuzzy Transportation Problem by Zero Point Method," Proceedings of the 15th Conference on Computer Science and Information Systems (FedCSIS), Sofia, Bulgaria, 2020, pp. 345-348. DOI: 10.15439/2020F61

[49] V. Traneva, S. Tranev, V. Atanassova, "An Intuitionistic Fuzzy Approach to the Hungarian Algorithm," in: G. Nikolov et al. (Eds.): NMA 2018, LNCS 11189, Springer Nature Switzerland, AG, 2019, pp. 1-9. DOI: 10.1007/978-3-030-10692-8_19

[50] V. Traneva, S. Tranev, M. Stoenchev, K. Atanassov, "Scaled aggregation operations over two- and three-dimensional index matrices," Soft computing, vol. 22, 2019, pp. 5115-5120. DOI: 10.1007/s00500-018-3315-6

[51] V. Traneva, S. Tranev, Index Matrices as a Tool for Managerial Decision Making, Publ. House of the Union of Scientists, Bulgaria; 2017 (in Bulgarian).

[52] V. Traneva, S. Tranev, "An Intuitionistic fuzzy zero suffix method for solving the transportation problem," in: Dimov I., Fidanova S. (eds) Advances in High Performance Computing. HPC 2019, Studies in computational intelligence, Springer, Cham, vol. 902. DOI: 10.1007/978-3030-55347-0_7, 2020 